\title{
Comunicación

\section{La Boletina de Morelia: órgano informativo de la Red Nacional de Mujeres, 1982-1985}

NATHALIE LUDEC*

El neofeminismo o la «segunda ola» del feminismo, de los años setenta entró en México por las puertas de los medios de comunicación. El presente análisis pone el acento en la experiencia de un grupo feminista de la provincia, el Colectivo VenSeremos, de Morelia, Michoacán, el cual dio origen de la Red Nacional de Mujeres. A través de su órgano informativo $L a$ Boletina, el grupo se esforzó por divulgar y confrontar las distintas experiencias de los grupos de mujeres de la República con la preocupación de difundir las ideas feministas. Rompió con el centralismo de la capital al proponer una difusión del feminismo, no sólo en sus dimensiones universales sino locales, con recursos propios de cualquier órgano alternativo.

PALABRAS CLAVE: feminismo, difusión, México, Michoacán, análisis de prensa.
Theneo-feminismor "secondwave» of feminism of the seventies entered Mexico through the gates of mass media. This research is focused on the experience of a feminist provincial group, the VenSeremos, from Morelia, Michoacan which originated the Red Nacional de Mujeres (Women National Net). Through its newsletter, La Boletina, this group strived to propagate and confront the different experiences of women groups all over the country in order to circulate the feminists views. La Boletina broke the capital city centralism when it decided to circulate feminism beyond its universal dimension, using local resources depending on any alternative newsletter.

KEY WORDS: feminism, diffusion, Mexico, Michoacan, press analysis.

* Universidad de París 8.

Correo electrónico: Nathalie.Ludec@univ-paris8.fr. 
«...Y usted, señorita, ¿qué hace?

- Dirijo el tráfico en la vía láctea...»

Alfonsina Storni

El neofeminismo o la «segunda ola» del feminismo, de los años setenta entró en México por las puertas de los medios de comunicación. Algunas publicaciones (Acevedo, 1970, 1972) de mujeres, que traducen textos de los movimientos norteamericanos y europeos, así como la serie radiofónica de Alaíde Foppa, ${ }^{1}$ Foro de la Mujer (1972-1980), dieron entrada a un raudal de nuevas ideas en un contexto político y económico favorable. El gobierno de Luis Echeverría Álvarez (1970-1976) impulsó la «apertura política» dentro de un marco económico próspero. El Distrito Federal se convirtió en el centro de recepción, de producción, de debates, con la creación de grupos y publicaciones feministas: La Revuelta (1976-1983), Cihuat (1975) y Fem (1976), que continúa viva hasta nuestros días.

Pero las teorías feministas, adoptadas más por convencimiento que necesidad, sólo interesan a una minoría de intelectuales y mujeres de la clase media. La mayoría de las mexicanas no se reconocen en las demandas de las feministas, que «extreman su audacia al exigir lo inconcebible: la despenalización del aborto» (Monsiváis, 2000:171). Tratar este tema como el de la violencia sexual en los medios masivos de comunicación era impensable.

El proyecto de ley sobre Maternidad Voluntaria es llevado por el Frente Nacional de Lucha por la Liberación y los Derechos de las Mujeres (FNALIDM), que funcionó entre 1979 y 1981, el cual reúne a grupos feministas, sindicatos universitarios, al Grupo LAMBDA de Liberación Homosexual y dos partidos políticos, al Partido Comunista Mexicano (PCM) y al Partido de las y los trabajadores (PTT). Dicho proyecto, presentado ante los diputados por los comunistas en 1980 , durante la presidencia de José López Portillo (1976-1982), fue atacado muy violentamente por el Comité Nacional Pro-Vida y la ultraderecha mexicana, y quedó archivado. Los grupos feministas en la ciudad de

1 Alaíde Foppa, guatemalteca exiliada en México por muchos años, fue una de las pioneras del feminismo latinoamericano y de la comunicación feminista. El ejército de su país la secuestró y asesinó en 1980 (Ludec, 2000). 
México salieron agotados de esta experiencia y atravesaron un período de reflujo (1983-1985); ni siquiera fueron capaces de organizarse para apoyar un proyecto de reforma del Código Penal sobre el aborto en 1983. Ante la reacción de la Iglesia Católica y la falta de apoyo de parte de los grupos feministas, el gobierno de Miguel de la Madrid retiró su propuesta.

Con todo surgieron nuevos grupos, dentro de un contexto de crisis económica, que afectó en particular a las mujeres de los sectores populares. Los grupos como Acción Popular de Integración Social (APIS, 1982); Grupo de Educación Popular con Mujeres (GEM, 1982); Centro de Investigación y Desarrollo Humano de América Latina (CIDHALMéxico, 1982), y Equipo de Mujeres en Acción Solidaria (EMAS, 1985), trabajan con las mujeres de los sectores populares, gracias a un financiamiento europeo. Esta tendencia del «feminismo popular de base» es representado principalmente por feministas socialistas, mujeres cristianas y ex militantes de los partidos de izquierda.

El contexto socioeconómico empujó a las mujeres hacia el mercado laboral, pero en los sectores menos calificados:

En el trabajo productivo la mujer ha incrementado su participación de manera evidente. Si en 1930 la población económicamente activa (PEA) femenina era apenas de un $4.6 \%$ de la PEA global, para 1980 ésta representa un $24 \%$ de la global. [...] De esta proporción, el 46\% trabaja en el sector servicios (en gran parte como empleadas domésticas), el $22 \%$ en el comercio y el $21 \%$ en la industria de transformación (Tuñón, 1987: 160).

Con todo, la regresión del analfabetismo femenino se refleja en la presencia mayoritaria de las mujeres entre los maestros: «Para 1980 las mujeres son $57 \%$ del total de maestros» (idem).

Esta nueva realidad laboral acarreó problemas sociales y familiares. Dentro de los grupos de mujeres, se debatió esta nueva situación que cuestionaba los roles sexuales dentro de la familia y la sociedad, así como las relaciones de pareja. De esta manera surgió una perspectiva feminista para tratar la violencia, las tareas domésticas, la libertad sexual.

Por toda la República, se constituyeron grupos con estas inquietudes, redes de comunicación, y se celebraron encuentros: entre 1980 y 1987 , 
hubo 10 encuentros nacionales y sectoriales (de colonas, 2 campesinas, trabajadoras). Mención especial merece la actividad de los grupos de provincia, por lo común marginados o invisibles en las discusiones y debates centralizados en la capital, y también en el sector académico de la investigación:«la historia del feminismo en México se cuenta desde la capital, lo cual la hace más bien historia del feminismo en la ciudad de México» (Colectivo VenSeremos, 2004).

El presente análisis pone el acento en la experiencia de un grupo feminista de la provincia, el Colectivo VenSeremos, de Morelia, Michoacán, al origen de la Red Nacional de Mujeres. A través de su órgano informativo La Boletina ( $L B)$, el grupo se esforzó por divulgar y confrontar las distintas experiencias de los grupos de mujeres de la República con la preocupación de difundir las ideas feministas.

El análisis temático del contenido redaccional, del número 3 (1983) hasta el número 6-7 (1984), que están en nuestra posesión, nos proporciona las herramientas para medir en qué medida La Boletina rompió con el centralismo de la capital al proponer una difusión del feminismo, no sólo en sus dimensiones universales, sino locales, con recursos propios de cualquier órgano alternativo.

\section{EL COLECTIVO FEMINISTA VENSEREMOS}

La iniciativa de organizarse en colectivo vino de cuatro mujeres de Morelia, estudiantes de filosofía en la Universidad Nacional Autónoma de México (UNAM). Su inquietud era «organizarse como mujeres en una población donde no había hasta entonces organizaciones de mujeres» (Colectivo VenSeremos, 1988). El 10 de mayo de 1982, se creó el Colectivo VenSeremos, en el cual participaron unas 30 mujeres; un mes después se constituyó la Red Nacional de Mujeres, que reunió a 21 grupos de todo el país. De los 21 grupos, la provincia cuenta con ocho (véase anexo 1). Su nombre es una invitación a ser colectivamente: «ven a ser, ven a nacer, ven seremos para después vencer» $(L B, 1983: 5)$. El Colectivo desarrolló

2 La ciudad de México se divide en barrios, llamados colonias, que se construyeron conforme iba creciendo la ciudad. Sus habitantes son los colonos y las colonas. 
sus actividades en el ámbito de las comunicaciones. Se hizo cargo de un programa radial, «Nosotras las mujeres», a partir de marzo de 1981. En agosto de 1983, abrió la columna dominical «Marginalia» en el diario matutino La Voz de Michoacán, el de mayor circulación en ese estado, con un tiraje de 50000 ejemplares (Medios publicitarios, 1988). Por último, el Colectivo VenSeremos se hizo responsable en enero de 1983, de la publicación La Boletina. Este boletín mimeografiado, modesto, alternativo, se planteó como un órgano de información y comunicación al interior de las organizaciones de mujeres, que conforman la Red Nacional de Mujeres de México.

\section{La Boletina}

La Boletina nació un día de junio de 1982, en el Distrito Federal, para después trasladarse a Morelia, hasta su último número en 1984.3

En un primer momento se decidió que la publicación se organizara en el Distrito Federal. Es así como dos grupos: el Grupo Autónomo de Mujeres Universitarias (GAMU) y el Grupo LAMBDA de Liberación Homosexual, se dieron a la tarea de sacar los dos primeros números. El primer número (1982) se financió con las cuotas de varios grupos. El segundo número (1982a) apareció en noviembre del mismo año para festejar el II Encuentro Nacional de Mujeres promovido por la Red (marzo de 1982).4 A partir del tercer número (1983), cuando La Boletina se trasladó a Morelia, se cumplió la voluntad de descentralizar algunas actividades del Distrito Federal. «Dentro de la Red, quedó decidido que algunos trabajos fueran rotativos, que ningún grupo se quedara con ellos por tiempo indeterminado, al contrario» (Colectivo VenSeremos, 1988).

3 Son dos las publicaciones con sede en México, DF (1982, 1982a) y cuatro las realizadas en Morelia, Michoacán (1983, 1983a, 1983b, 1984) . Para 1985, el grupo de Colima tenía que seguir con la publicación, pero se interrumpió el proyecto sin saber las causas, según Anabela Rodrigo (Colectivo VenSeremos, 1988).

4 El I Encuentro fue organizado en febrero de 1981, por GAMU, al cual asistieron cerca de 17 grupos del movimiento feminista con diversas orientaciones (Grupo Cinco, 1991:147). 
El Colectivo VenSeremos de Morelia se encargó de la publicación de cuatro números (1983, 1983a, 1983b, 1984), con una frecuencia discontinua: «La publicación era autofinanciable, los grupos pagaban cuotas por adelantado, para la compra del papel, la impresión, lo más caro, y el envío a toda la República» (idem). El tiraje era de unos 3000 ejemplares. ${ }^{5}$ Cada grupo se comprometía en comprar entre 50 y 100 ejemplares, según su capacidad de distribución, en 30 pesos la unidad.

Fundamentalmente, son dos mujeres las encargadas de recopilar material, organizarlo y estar pendientes de su tiraje y distribución, por correo o directamente. Diagraman La Boletina, que aparece en forma rudimentaria.

Yo soy la persona que en Morelia se encargaba de la producción de La Boletina, junto con Anabel Rodrigo. El trabajar en la Secretaria de Educación en el estado de Michoacán me facilitó conseguir el apoyo del secretario (Ing. Diodoro Guerra) y la impresión en offset se realizaba en los talleres de la Secretaría. También nos proveían el papel. Nosotras luego nos encargábamos de compaginar, engrapar y enviar los ejemplares a los grupos de mujeres en todo el país, con nuestros propios recursos y trabajo (Entrevista con Josefina María Cendejas).

La publicación tiene un formato modesto $(20$ x17 cm), el papel es de baja calidad, el texto apretado dentro de la compaginación, a veces las letras son borradas, las ilustraciones en blanco y negro, los dibujos a lápiz, las reproducciones de fotografías y cuadros son escasos, concentrados en las portadas. El número de páginas, sueltas, pasó de 16 (1983) a 36 (1984). El contenido no se reparte según secciones fijas, excepto la última página que informa sobre las manifestaciones feministas (marchas, campañas, jornadas, mitines, encuentros, actividades artísticas) bajo el título «+ Claves y + Clavos». El público de La Boletina está constituido principalmente por las mujeres organizadas de la Red, pero también «se distribuye a otras mujeres y hombres solidarios o simplemente interesados» (ILET, 1984: 21).

5 El tiraje de la mayor publicación feminista de aquel entonces, Fem, era de 6000 ejemplares. 
Por sus características físicas y su organización interna, La Boletina pertenece a la comunicación alternativa de la mujer, una seña de identidad de la difusión del pensamiento feminista que quedó al margen de los medios masivos de comunicación.

\section{LA BOLETINA: UN MEDIO}

DE COMUNICACIÓN ALTERNATIVA

La comunicación alternativa de la mujer aspira a «constituirse en un instrumento educativo, solidario y participativo» y a fortalecer los movimientos sociales (Santa Cruz, 1984: 5).

\section{«La Boletina es de todas»: participación y solidaridad}

Esta declaración, que funciona como lema, se sitúa en la contraportada de la publicación y significa la elaboración colectiva del contenido a cargo de los distintos miembros de la Red: «iEnvíanos material para su publicación: denuncias, reflexiones, información, testimonios, poemas, dibujos, fotografías, todo lo que se te ocurra!».

La publicación número 3 (1983), la primera que quedaba a cargo del Colectivo VenSeremos, se centró en la información sobre las características y actividades de unos grupos de mujeres que conforman la Red: el Grupo de Mujeres de Xalapa (Veracruz); Comunicación, Intercambio y Desarrollo Humano en América Latina (CIDHAL) 6 de Cuernavaca (Morelos); el Colectivo VenSeremos; el Grupo Mujeres de Culiacán (Sinaloa); el Centro de Apoyo para Mujeres Violadas y Golpeadas (CAMVAG) de Colima (Colima). La existencia de La Boletina era una necesidad para los pequeños grupos de la provincia, como lo afirman las cuatro mujeres de Jalapa: «La idea de La Boletina nos pareció excelente porque nos hemos sentido abandonadas y aisladas en la provincia [...] ¡Apoyémonos!» $(L B, 1983: 2)$. El motor de esta aglutinación dentro de la Red fue el II Encuentro de Mujeres de México, en marzo de 1982.

La participación en La Boletina está abierta a todas las mujeres que lo deseen. Es así como no sólo mujeres de la Red sino muchas otras han enviado testimonios de su quehacer, de sus vivencias. Una «trabajado-

6 http://www.laneta.apc.org/cidhal 
ra, ama de casa y madre de familia», de 31 años, del estado de Quintana Roo, «el estado más alejado y fregado», manda una carta donde se queja de la carestía de la vida, la falta de infraestructuras, la escasez de relaciones sociales, por el machismo y el alcoholismo en su pueblo, y se propone participar en algo para mejorar la situación (ibid.:15).

La Boletina es un lugar de encuentro entre los grupos que se enteran, ahí precisamente, de la actualidad feminista: elabora balances de los encuentros, como el del III Encuentro de la Red Nacional de Mujeres, realizado en Colima, en abril de 1983 (1983b:2-4), o el del II Encuentro Feminista Latinoamericano y del Caribe, celebrado en Lima en julio de 1983 (ibid::6-8). Fortalece un vínculo de pertenencia a una misma filosofía, alrededor de fechas simbólicas como el 8 de marzo, Día Internacional de la Mujer, festejado en varias partes de la República: Sinaloa, Cuernavaca, Morelia y el Distrito Federal. La participación de las propias mujeres en la elaboración de la publicación significa una construcción conjunta de un proceso emancipador, el cual empezó en el siglo XIX.

\section{FUNCIÓN EDUCATIVA}

La composición gráfica de las portadas remite a la historia de la mujer en su lucha por su emancipación. Valoriza a la mujer como sujeto histórico, protagonista de su destino. En la portada del tercer número (1983), un grupo de tres mujeres, vestidas según la moda del siglo XIX, pero sin semblante -no son ciudadanas-, hacen punto juntas y sus hilos se mezclan para escribir una ideas del pensamiento feminista, entre otras: «liberación», «organización», «solidaridad», «sin oprimirnos», «mujeres libres», «un mundo donde podamos vivir juntos». La labor femenina, revalorizada, remite a la acción común, solidaria y contestataria, fructífera de las mujeres organizadas en redes. La escena será una referencia al origen de la prensa para mujeres en el siglo XIX que se empeñó en desarrollar la educación, cuyo acceso permitió a las mujeres incursionar en el periodismo. En el semanario El Álbum de la Mujer (1883-1890), la escena será una referencia al origen de la prensa para mujeres en el siglo XIX. En el semanario, fundado por la periodista española Concepción Gimeno Flaquer, en 1883, se preguntaban: «¿Es acaso incompatible coser y razonar?» En la portada del número 4 
(1983a), un dibujo a lápiz de niño, pone en escena a una maestra ante la pizarra, siendo el sector educativo un espacio público conquistado por las mujeres, también desde el siglo XIX. Si seguimos esta lectura de las primeras planas, acabamos con el retrato de una joven de los años ochenta, sonriente, resplandeciente, que trasmite confianza y bienestar.

Más directamente, La Boletina publica análisis feministas sobre la sociedad y la situación de la mujer según distintos enfoques que la publicación define: «marxista» (1983:14), «antropológico estructural» (1983a:11-12) y «biologicista» (ibid::14-16).

La perspectiva marxista es la que predomina. En un artículo titulado «No basta saber que estamos oprimidas», anónimo, se interpela a las amas de casa que tienen que luchar contra el capital que «se apropia de su trabajo»: en el sistema capitalista «el trabajo doméstico produce la fuerza del trabajo [...] sin recibir pago». Además, las amas de casa tienen cada vez más dificultades para cumplir estas tareas en período de crisis económica, 7 cuyo responsable es el «PRI gobierno»8 (1983a:4). La crítica ofrece dos vertientes: lo privado adquiere una dimensión política. En el número siguiente (1983b:14), el Colectivo de Torreón (Coahuila) profundiza el «análisis feminista» de la «opresión de la mujer», más bien de la trabajadora, la obrera, que es doble, es una dominación de una clase por otra y de un sexo por otro. El feminismo sufragista ya cumplió con su cometido, ${ }^{9}$ ahora el feminismo tendrá una «concepción revolucionaria que rebase el feminismo puramente teorizante y el feminismo resentido» (idem).

Se esbozan aquí las etapas de la expresión del movimiento feminista: en una primera etapa, se trató por supuesto de entender y asimilar

7 De hecho, la crisis económica se manifestó abiertamente a partir de 1982, con un proceso inflacionario creciente, una deuda externa de unos 108 millones de dólares, una fuga de capitales creciente. La crisis económica, aunada a la política, de austeridad implantada por el gobierno, provocó un deterioro de las condiciones de vida de las familias, afectadas por el aumento de la tasa de desempleo y la caída del salario real (Navas et al. 1987:138-139).

8 El Partido Revolucionario Institucional (PRI) emanado de la Revolución es el gobierno. Tal hegemonía cesó con las últimas elecciones presidenciales de julio de 2000, que dieron la victoria al candidato del Partido de Acción Nacional (PAN), Vicente Fox.

9 Las mexicanas obtienen el voto en 1953. 
las teorías feministas, sobre todo cuando venían de fuera. Pero se le reprochó a las feministas su tono victimario, quejumbroso, al fin y al cabo contraproducente. Pero a partir de los años ochenta, el feminismo presenta otra cara: el feminismo popular de base coincide con la orientación socialista del feminismo. Ésta comparte la idea de la lucha de clases, es anticapitalista y antiimperialista. Sus temas predilectos son el estatuto teórico del trabajo doméstico, la participación de las mujeres en la producción económica, el empleo femenino, los procesos de reproducción de las desigualdades sociales.

Ocupar el espacio público cuestiona las estructuras sociales y mentales. Dentro de una perspectiva «antropológica estructural», con referencias a la obra de Levi-Strauss, en un artículo titulado «Cuando el signo habla», Josefina María Cendejas, del Colectivo VenSeremos, propone, de parte de la mujer, una conquista y una valoración de la palabra, que cae bajo el dominio masculino ( $L B, 1983 \mathrm{a}: 11-12)$. El discurso femenino suele ser tachado de frívolo, espacio de la intriga, del chismorreo. Vencer el prejuicio significa crear nuevos espacios de sociabilidad, en todos los ámbitos, cultural, político y económico: en una inversión de connotaciones las pancartas enarbolan durante la jornada del 8 de marzo el refrán misógino, «¿cabellos largos, ideas cortas?».

Ciertas investigaciones científicas dan pábulo a unas interpretaciones abusivas sobre la superioridad del cerebro masculino. La Boletina denuncia el contenido de un artículo publicado en el periódico Unomásuno, titulado «El cerebro femenino es diferente al del hombre», que refuerza «cierta ideología sexista», base de la «estructura patriarcal» de la sociedad. La autora, doctora Leticia Quesnel Galván (GAMU), se vale de trabajos de la socióloga francesa Evelyne Sullerot para desmontar «la argumentación biologicista» y se levanta contra la «popularización» de este tipo de argumentos en un media nacional (ibid.:14-16).

La Boletina es un espacio de divulgación para las tesis feministas y para la formación teórica; dos funciones alimentadas por reseñas de libros cuyas referencias reflejan el origen extranjero del pensamiento feminista (véase anexo bibliográfico). Se puede repartir los libros según las categorías siguientes: Historia (2), Feminismo y socialismo (2), Feminismo y lesbianismo (1), Feminismo radical (1), Cuerpo (1), Psiquiatría (1), Feminismo y México (1), Literatura femenina en México (2) 
(1983b:15; 1984:31). De las 11 referencias, sólo cuatro son mexicanas. Se destaca la preferencia por un femenismo socialista y radical, ${ }^{10}$ que responde más al contexto mexicano. En realidad, se produjo un rechazo del feminismo burgués o liberal,11 exportado de Europa y Estados Unidos, aunque fuera la primera expresión del feminismo mexicano a principios de los años setenta.

La labor educativa de La Boletina plantea los logros, avances y luchas pendientes de los grupos feministas, cuyos métodos de trabajo expone de manera didáctica.

\section{FUNCIÓN LIBERADORA}

La base organizativa del feminismo fue el pequeño grupo de «autoconcientización», de «auto-estima» ( $L B, 1983)$. Esta práctica se observa en todos los grupos feministas que organizan conferencias, elaboran cursos y trabajos prácticos, sobre temas como, por ejemplo, la salud para mujeres, el cuerpo y de ahí la sexualidad o el trabajo, las tareas domésticas y al final la doble jornada. Estas actividades se sitúan en nuevos espacios de sociabilidad para las mujeres, será una librería, una cafetería o un taller. La experiencia individual adquiere una dimensión colectiva. La Boletina describe la experiencia del Ta-

10 «El feminismo radical considera la lucha socialista condición necesaria pero no suficiente para el establecimiento de una sociedad en las que las mujeres sean libres. El socialismo se supone que no incluye el feminismo, mientras que el feminismo sí puede contener el socialismo. Marcuse reconoce que 'también las instituciones socialistas pueden discriminar a la mujer' y que en este sentido 'no sólo está justificado sino que es necesario un movimiento de mujeres independiente'» ( Sau, 1981:113).

11 «Es reformista y no revolucionario. Promueve los valores individuales, quiere reducir las desigualdades. Se conforma con conseguir para las mujeres las mismas oportunidades que los hombres sin cuestionarse el modelo socioeconómico vigente. Creen que cuando todas las mujeres trabajen como los hombres y en los Parlamentos el porcentaje de diputados esté a 50\% (como es la proporción de hombres y mujeres en la sociedad), las cosas marcharán bien. No se plantean las diferencias de clase ni el imperialismo ni el modo de producción capitalista... » (Sau, 1981:112). 
ller del Chopo, 12 un grupo del Distrito Federal, que trabaja sobre la «autoimagen», en grupos de seis mujeres. La sesión se desarrolla a partir de un trabajo manual, la confección de una figura que será una «autorepresentación» de cada participante. Este trabajo introspectivo permite liberar la palabra y la conciencia, y se propone crear las condiciones para debatir sobre los condicionamientos culturales y sociales $(L B, 1983: 7-9)$ y llegar a cuestionar la inamovilidad y el carácter «natural» de muchos de los roles femeninos (1983a:6-8). Las mujeres interpretan a las muñecas de trapo, que traducen desprecio, desvalorización, timidez, represión o libertad, insatisfacción o frustración. Los objetos desembocan sobre otro tema, el de la niñez - «nuestro presente está muy determinado por lo que fue nuestro pasado»- que conlleva los juegos, la percepción del adulto, de los padres. Es el propio grupo quien saca las conclusiones y no discursos teorizantes:

El rol femenino, marcado a fuego desde pequeñas en la familia y en la escuela, a través de la vestimenta, los juegos, las tareas domésticas, los mensajes de los medios masivos de información, van moldeando nuestras expectativas (1984:10-13). ${ }^{13}$

En esta labor de entender y valorar la vida de las mujeres, La Boletina anuncia regularmente las actividades del Colectivo Cine Mujer, ${ }^{14}$ a través de «Zafra AC, distribuidora de cine independiente, [que] ofrece un ciclo de películas elaboradas por mujeres», más bien documentales sobre «la represión en la escuela, el hogar, la violación» (1983b:20). La última película versa sobre el trabajo doméstico de las mujeres de las «vecindades», 15 le da visibilidad y valor (Vida de ángel) (1983a:1). Rehabilita así

12 El Taller del Chopo se inició en 1980-1981, como un programa de talleres sobre sexualidad, al que asisten todo tipo de mujeres, entre ellas, maestras, enfermeras, amas de casa, empleadas y estudiantes (Fem, 1983:57).

13 Estos tipos de talleres siguen existiendo hoy en los Centros de Atención a la Mujer (CAM) en las colonias populares del Distrito Federal.

14 El Colectivo Cine Mujer surgió alrededor de 1975. Una de las primeras películas que se hicieron versó sobre el aborto. En 1983 se hizo Mujeres yalaltecas, que se filmó en la Sierra zapoteca de Oaxaca (Hiriart, 1984:39-40).

15 La vecindad es un edificio repartido en alojamientos populares de una pieza cada uno o un conjunto de alojamientos distribuidos alrededor de un patio. 
la imagen de la mujer del sector popular urbano que tiene que enfrentarse con «los caseros», «los casatenientes».

La vida para ellas no es, a pesar de las enormes dificultades, un martirio, un camino de permanente enajenación, de sordidez, de brutalidad, como suele propogandizarse a través de los medios de comunicación (ibid.:2).

Gracias al flujo participativo de la información, horizontal, desde y con las mujeres, La Boletina contribuye a democratizar las comunicaciones, facilitando las relaciones entre las mujeres, así como los vínculos entre la capital y la provincia. Participa de la comunicación alternativa de la mujer que

quiere recoger el malestar de muchas mujeres, su insatisfacción, sus contradicciones y sus angustias, no para canalizarlos hacia el consumo, sino hacia una mayor lucidez que la mueva a participar críticamente, en el logro de cambios positivos en su beneficio y en beneficio de la sociedad en su conjunto (Santa Cruz y Erazo, 1983: 86).

\section{TAREAS Y MENSAJES DE LA BOLETINA:}

\section{LOS TÓPICOS DEL FEMINISMO}

La Boletina se plantea como «expresión de un proyecto histórico de cambio, de resistencia cultural y de construcción solidaria» (idem).

\section{Resistir}

El cambio pasa por la subversión - «la capacidad subversiva del feminismo» ( $L B, 1983: 5)$ - , primero del lenguaje y luego de los modelos y roles sociales, a partir de una conciencia crítica y creadora. La publicación aboga por «la liberación de cada una de nosotras como la subversión de todo lo establecido» (1983a:13).

Su mismo nombre -La Boletina- subvierte de entrada las horribles separaciones de género en las cuales la mujeres siempre perdemos, inmersas como estamos en un lenguaje masculinizado (Colectivo VenSeremos, 1988). 
Resulta que el contenido se ve salpicado de neologismos - «jornada marziana» (LB, 1983:6), «miembra» (1984:25). Esta actitud de entrar en resistencia, en el plan lingüístico, acarrea un estilo un tanto pesado cuando insisten, por ejemplo «en el descentramiento [y descentralización de poderes] y en la desestructuración de la jerarquía piramidal» (ibid.:9). Por otro lado, la expresión y las figuras de estilo son otras piezas de un sistema cerrado y remiten al ámbito de la procreación: las «Hijas de $\mathrm{La}$ Boletina» (1983b:2) son «sembradoras de inquietud, de inconformidad, de cambio», en el II Encuentro Feminista Latinoamericano y del Caribe, en Lima (ibid.: 6). El número 6-7 (1984) se organiza alrededor del tema de la niñez y de la niña.

Todo ello participa del «mujerismo», 16 del sisterwood anglosajón o de la sororité francesa, de una lógica amorosa entre las mujeres. Así La Boletina llama a la unión de «todas las mujeres» como si constituyeran una clase social oprimida (1983a:5), a la «solidaridad entre mujeres, por encima de las diferencias de credo, raza y clase social» (1983b:8). El discurso militante pretende unir a las mujeres, en particular a las amas de casa, en una clase social homogénea (1983a:4). El «nosotras» está omnipresente en el discurso, siendo los artículos raramente firmados.

Esta actitud «mujerista» explica el rechazo de la doble militancia por una parte de las feministas (1984:8-9). Primero, son los partidos de izquierda los que ofrecen más convergencia con el credo político feminista, pero dentro de los partidos se reproduce «el esquema jerárquico de dominación», convirtiéndose la militante en «la reina del mimeo»o de la cafetería. «La mujer, dentro del partido, en lo que respecta a mis observaciones personales, se caracterizó por ser de tipo accesorial y secundario y no de una real participación» (ibid:20-22).

Pero, ¿dónde debatir sino en los órganos institucionales, so pena de hacer mucho ruido por nada? Los riesgos son la asfixia y la ineficacia de los pequeños grupos encerrados en su utopía. Cabe señalar, con todo, lo fructífero de esta solidaridad «mujerista» en el apoyo mutuo entre

16 El mujerismo es una concepción que esencializa el hecho de ser mujer, idealiza las condiciones «naturales» de las mujeres y mistifica las relaciones entre mujeres. Una típica actitud mujerista es hablar en nombre de las mujeres, como si éstas tuvieran una posición uniforme en la sociedad. 
los grupos, cuando por ejemplo, se trata de salvar a la revista Fem de la bancarrota, al suscitar nuevas suscripciones (ibid::10).

Este afán furioso de identificarse, con un registro militante, una jerga propia, es un rasgo común de los movimientos sociales contestatarios. En el caso del feminismo, esta actitud dio pábulo a críticas acerbas, distorsiones, burlas y ridiculización, en los medios masivos de comunicación. Las palabras feminismo-feminista siguen teniendo mala prensa.

\section{Criticar}

El feminismo no es unidimensional; el que se expresa en La Boletina refleja la corriente radical, afirmando una demanda fundamental, piedra angular del movimiento de liberación, el derecho al aborto, que moviliza a todas las feministas. Además, el examen de las características de los grupos de la Red revela una preferencia por todos los temas que conciernen el cuerpo, objeto de atención, de observación, y por otra parte, de violencia.

La libertad sexual significa el acceso a los medios anticonceptivos, que remite a los «derechos reproductivos», la legalización del aborto, el fin de la estigmatización de la homosexualidad, al fin y al cabo, el rechazo de un determinismo moral y biológico que le niega a la mujer el derecho sobre su propio cuerpo.

Defender los derechos reproductivos ${ }^{17}$ de la mujer adquiere una dimensión internacional con el grupo International Contraception, Abortion and Sterelisation Campaign (ICASC), que «se formó en 1977 a raíz de una conferencia internacional en París, de feministas socialistas» (LB, 1983b:5). En 1981 se reunió de nuevo el Grupo y para 1984, se prevé, en México

un Encuentro Internacional de Denuncia sobre los abusos contra la mujer que se realizan en la esterilización, los anticonceptivos y el aborto [...] para que

17 En los años ochenta el concepto de derechos reproductivos es asumido por un gran número de feministas en el mundo, ya sea en el discurso académico o en las campañas y demandas internacionales. Las bases ideológicas del término se encuentran en « los conceptos de integridad corporal y autodeterminación sexual, característicos del feminismo de la segunda ola» (Correa y Petchesky:1994). 
hiciera contraste con el Congreso Internacional de Población que se llevará a cabo en nuestro país en el año 1984, y como una respuesta de las mujeres al mismo (idem).

Será un tribunal Encuentro. Ocurre que las políticas de población se organiza a expensas de las mujeres, convertidas en conejillos de Indias: en los años ochenta, el uso de la inyección anticonceptiva (Depoprovera) no fue aprobado por las autoridades norteamericanas y, sin embargo, ha sido utilizada en ochenta países», entre los cuales está México (Fem, 1988:18). Pero La Boletina parece olvidar el principio de precaución cuando anuncia, de manera sensacionalista un supositorio abortivo, el Preglandin, de origen nipón, retomando la noticia publicada en el periódico nacional Unomásuno ( $L B, 1984: 25)$, objeto de sus críticas en un número anterior (1983a:14-16), por su falta de rigor científico.

Ahora bien, la Red Nacional de Mujeres se propuso coordinar el Encuentro Internacional de la Mujer sobre los derechos reproductivos, al centralizar la información en el CIDHAL, en Cuernavaca. Todo el aspecto material quedó bien organizado, encontrar locales para los debates, prever el hospedaje, comidas, guarderías, traducción simultánea, pero de los «50 grupos diferentes de mujeres y grupos de salud popular», ninguno respondió y el Encuentro se canceló:

Nos dimos cuenta que en nuestro propio país no habíamos logrado a todo lo largo del año 1983 propiciar la discusión sobre los anticonceptivos, el aborto o la esterilización (1984:3-4).

Esta desgraciada aventura muestra la permanencia de temas tabúes y resistencias dentro de la sociedad mexicana, que se llaman la familia, la Iglesia Católica, los partidos políticos, en suma, «el conjunto denomido 'moral y buenas costumbres'» (Monsiváis, 2000: 170). Por otro lado, recordemos el fracaso del proyecto de ley sobre Maternidad Voluntaria, en 1980, y el de reforma del Código Penal sobre el aborto en 1983, que no condujeron a ningún avance respecto a la despenalización del aborto. A pesar de todo los grupos feministas se empeñan en reclamar para la mujer «el derecho sobre su propio cuerpo» $(L B, 1984: 7)$ : el 
grupo de Culiacán, en marzo de 1982, apareció «públicamente en una mesa redonda sobre la legalización del aborto» (1983:6).

Al fin y al cabo, la meta final es

una sociedad sin abortos, donde no haya que andarse debatiendo entre la prohibición y el pecado, porque las mujeres podrán decidir sobre su vida y se tendrán los medios necesarios y eficaces al alcance (1984:8).

Señalemos cómo las feministas trasformaron el discurso del aborto, percibido como crimen, en un concepto mucho más amplio que denota el derecho humano de las mujeres a la autodeterminación sobre su propia fertilidad, su maternidad, y los usos de sus cuerpos. El concepto se trasformó para acercarse a las preocupaciones democráticas. La Boletina denuncia la intromisión de la moral en los asuntos sexuales que entran en la esfera personal. Aboga por cualquier orientación sexual, desvinculada de la procreación, abriendo sus páginas a los grupos lésbicos (LAMBDA, OIKABETH). 18

Las demandas expresadas en La Boletina se vinculan con una trasformación de las relaciones en la pareja, en el hogar y en la sociedad. Los objetivos de los grupos de la Red son comunes: «una transformación profunda de las estructuras de nuestra sociedad en todos los niveles: socioeconómico, ideológico y político» (1983:3) o «un cambio estructural de esta sociedad patriarcal», cuya violencia machista e institucional se ejerce en toda impunidad en un régimen en suma no democrático (ibid::6). Unos centros de mujeres sufrieron de la represión policíaca: el gobernador del estado de Morelos, Lauro Ortega, mandó cerrar el Centro de Documentación, la Biblioteca y el Centro de Salud de CIDHAL, «uno de los bastiones de lucha del movimiento de mujeres» (1984:2), y también el local de trabajo y la casa de mujeres del grupo Lesbianas Morelenses, «sometiéndolas a una constante vigilancia y

18 En 1977 se forma el primer grupo de lesbianas, cuyo nombre fue Lesbos. A ése le siguieron OIKABETH I (Organización Autónoma de Lesbianas Feministas) en 1978; Lesbianas Morelenses, en 1980; OIKABETH II, Cuarto Creciente y Oasis Morelense, en 1983 (OpusGay, 2004). 
persecución policíaca» (ibid.:36). Estos actos suceden en un contexto de seudo «apertura» democrática del gobierno priísta. 19

Mención especial merecen unos avances concretos para combatir la violencia contra las mujeres. La acción del gobierno de Colima es ejemplar. La gobernadora del estado de Colima, Griselda Álvarez, ${ }^{20} \mathrm{al}$ origen del «Reglamento del Centro de Apoyo para Mujeres Violadas y Golpedas» (CAMVAG), fue una pionera en el intento de institucionalizar la atención y la ayuda a las mujeres violadas y golpeadas. El CAMVAG «es una dependencia de la Procuraduría General de Justicia del estado de Colima» (LB, 1983:12).

La primera gobernadora que tuvo México recuerda los cambios que impulsó en el Código Penal para que ninguna mujer en Colima diera a luz en la cárcel o bien para que las mujeres mayores de 75 años, que cometieran algún delito, cumplieran su sentencia en arresto domiciliario. ${ }^{21}$

Pero siguen expresándose públicamente los antifeminismos y las homofobias, hasta en el pionero estado de Colima.

\section{Crear}

El arte permite trascender los cuestionamientos y las demandas feministas, que siguen incumplidas. Lo inalcanzabe se realizará de momento dentro del espacio de la imaginación, del sueño, de la utopía, en nuevos contenidos culturales. El contenido cultural de La Boletina, que cubre varios géneros, poesía, literatura, teatro, esboza un sujeto femenino que refleja las opciones de un feminismo cultural, que crea una nueva «palabra de mujer». Esta cultura valora el ser mujer en su propio cuerpo, se enraiza en la naturaleza y la intimidad del cuerpo como este poema de

${ }_{19}$ El Frente Nacional contra la Represión (FNCR) contabiliza « 38 desaparecidas entre los 502 presos políticos no reconocidos en México» ( Grupo Cinco, 1991:11).

20 Literata, poetisa, editorialista, Griselda Álvarez Ponce de León fue senadora por Jalisco de 1977 a 1979 y electa gobernadora de Colima a los 66 años de edad (Cervantes, 2003).

${ }^{21}$ Silvia Magally, «Cuatro mujeres gobernadoras en México», http://www.proceso.com.mx/exclusivas.html?eid $=2070$ 
la escritora mexicana Rosa María Roffiel, «Mi vulva es una flor» / «Mi vulva es una flor, es una concha, un higo, un terciopelo» ( $L B, 1983: 10)$, que se destaca en la literatura lésbica. Se valoriza la «diferencia roja» en esta «herida / destinada eternamente a sangrar» (1983b:12). La publicación ofrece textos poéticos de autoras nacionales y extranjeras, «Entre nosotras», de Ángeles Mastretta, Patricia Rodríguez, Moravia Ochoa, Katherine Mansfield, Marge Piercy (1984:13,15-19). Esta última expresa el deseo de maternidad frustrado en la pareja lésbica:

No podemos tener hijos.

[...]

Cuando pasen los años habremos de jugar a que tenemos hijos.

Podemos amarnos. Amarnos. Amarnos (ibid.:19).

Las mujeres de la organización lésbica OIKABETH, «guerreras que abren camino y esparcen flores» sueñan con la «liberación de la humanidad», una sociedad sin clases «ni siquiera en las escuelas», en un comunicado imaginado para el «año 20 001» (1983a:9). El concepto de «guerreras» en el lenguaje de los grupos lésbicos revela la situación conflictiva que viven, según Roffiel, «aquellas que por tener una preferencia sexual distinta a la heterosexual, son rechazadas y estigmatizadas».22

La publicación se convierte en un medio cultural que privilegia la expresión femenina. En una pieza de teatro en un acto, Asfixiata, de Fernanda Navarro, del Colectivo VenSeremos, expone en una entrevista para un diario nacional, Cataclismo, cuatro hitos del movimiento feminista llamado «La costilla prófuga», con reminiscencias bíblicas. Primero se rebela contra su estatuto de «esposada», en realidad desposada, también de «mujer domesticada» al mismo tiempo florero, totalmente dominada por la autoridad del marido. Por otro lado su militancia política, «en la sinistra, o siniestra», la deja desilusionada. Lucha contra los estereotipos divulgados por los medios de «incomunicación masiva», que imponen un comportamiento uniformizado con una única referen-

22 http://www.jornada.unam.mx/1999/08/02/breves.htm\#libr_lesb 
cia, el hombre. Finalmente, el espacio de su lucha se llama «UTÓPIKA, Michoacán» (LB, 1983b:17-19).

La Boletina construye un espacio específicamente femenino, endogámico, efímero por definición, sólo posible en el ámbito de la utopía, de la creación, donde está a sus anchas la capacidad subversiva del feminismo.

El discurso de La Boletina refleja la ideología de un movimiento que busca unas señas de identidad nacionales y que apenas se empieza a entender como un instrumento de lucha y no sólo como un instrumento de análisis. Plantea también todos las cuestiones claves de las prácticas, de alcance político, de las feministas que temen finalmente la cooptación. Pero participar en las instituciones significa fortalecer la democracia, valiendo las propuestas feministas, tanto para los hombres como para las mujeres. El feminismo avanza como «atmósfera de reconsideración del comportamiento de las mujeres y de rectificación social y política» (Monsiváis, 2000:203).

El Colectivo VenSeremos y La Boletina cumplieron con el objetivo de crear una red de comunicación alternativa entre la capital y la provincia. Redujeron el aislamiento y la dispersión de los grupos feministas de la República. Desgraciadamente la experiencia terminó apagándose por el desgaste que representan las condiciones de existencia de un medio de comunicación alternativa como el trabajo voluntario, la escasez de recursos y apoyos económicos o la falta de experiencia de las mujeres, lo cual no significa que no siga intentándose este camino, como la publicación trimestral La correa feminista creada en 1991 y editada por el Centro de Investigación y Capacitación de la Mujer (CICAM) del Distrito Federal, que ha tendido puentes entre los estados y la capital (CICAM, 1992). En el número 19, otorno-invierno de 1998, anuncia su forma virtual a través de Creatividad Feminista. ${ }^{23}$ De hecho, las redes de comunicación electrónica, como Comunicación e Información de la Mujer AC24 en México, el portal de la mujer latinoamericana MujeresHoy, 25 o el sitio electrónico de Isis Internacional26 vencen las distancias e ignoran las fronteras.

\footnotetext{
$23 \mathrm{http}$ //creatividadfeminista.org

24 www.cimacnoticias.com

25 www.mujereshoy.com

26 www.isis.cl
} 
ANEXO 1

GRUPOS FEMINISTAS PERTENECIENTES

A LA RED NACIONAL DE MUJERES

Grupos del DF:

- Acción Cívica Feminista AC

- Centro de Apoyo a Mujeres Violadas, aC (CAMVAC)

- Grupo Acratas

- Grupo Autónomo de Mujeres Universitarias (GAMU)

- Grupo LAMBDA de Liberación Homosexual

- Grupo de Mujeres

- Grupo de Mujeres de Trabajo Social

- Lucha Feminista

- Movimiento de Liberación de la Mujer (MLM)

- Movimiento Nacional de Mujeres (MNM)

- Organización Autónoma de Lesbianas Feministas (OIKABETH)

- Revuelta

- Taller del Chopo
Grupos de los estados:

- CIHUATL (mujer en náhuatl), (Monterrey, Nuevo León)

- Colectivo Autónomo Feminista (CAFEM) (Saltillo, Coahuila)

- Colectivo Feminista de Colima, AC (COFEMC)

- Colectivo Nosotras (Guadalajara, Jalisco)

- Colectivo VenSeremos (Morelia, Michoacán)

- Emancipación (Tijuana, BC)

- Grupo Autónomo de Mujeres (GAM) (Torreón, Coahuila)

- Grupo de Mujeres (Durango, Durango)

\section{ANEXO 2 \\ SIGLAS DE ORGANIZACIONES FEMINISTAS}

*APIS: Acción Popular de Interación Social

CAFEM: Colectivo Autónomo Feminista

CAM: Centros de Atención a la Mujer

CAMVAC: Centro de Apoyo a Mujeres Violadas AC

CAMVAG: Centro de Apoyo a Mujeres Violadas y Golpeadas

CICAM: Centro de Investigación y Capacitación de la Mujer

*CIDHAL: Centro de Investigación y Desarrollo Humano de América Latina

COFEMC: Colectivo Feminista Colima

"EMAS: Equipo de Mujeres en Acción Solidaria

FNALIDM: Frente Nacional de Lucha por la Liberación y los Derechos de las Mujeres
FNCR: Frente Nacional contra la Repreción

GAM: Grupo Autónomo de Mujeres

GAMU: Grupo Autónomo de Mujeres Universitarias

*GEM: Grupo de Educación popular con Mujeres

ICASC: International Contraception, Abortion and Stevelisation Campaing

*MAS: Mujeres en Acción Solidaria

MLM: Movimiento de Liberación de la Mujer

MNM: Movimiento Nacional de Mujeres

OIKABETH: Organización Autónoma de Lesbianas Feministas

Organizaciones pertenecientes al Grupo Cinco. 


\section{ANEXO 3}

OTRAS ORGANIZACIONES FEMINISTAS MENCIONADAS

- Colectivo Cine Mujer

- Colectivo Nosotras

- Cuarto Creciente
- $\quad$ Grupo de Mujeres (DF, Jalapa, Durango, Culiacán)

- Lesbianas Morelenses

- Oasis Morelense

ANEXO BIBLIOGRÁFICO:

REFERENCIAS A VECES INCOMPLETAS EN LA BOLETINA

Basaglia, Franca y Dora Kanoussi, Mujer, locura y sociedad. Puebla: Universidad Autónoma de Puebla.

CiDHAL, Centro de Investigación y Desarrollo Humano de América Latina (1983) Cuerpo de mujer. Cuernavaca, México: CIDHAL.

Cuentistas mexicanas, Antología. México: UNAM, Literatura de Mujeres.

KRAUZE, Ethel, Intermedio para mujeres. México: Océano.

LiNNHOFF, Úrsula (1983) La homosexualidad femenina. Col. La educación sentimental, 7. México: Anagrama.

LONZI, Carla (1983) Escupamos sobre Hegel. México: Anagrama.

MiCHEL, Andrée (1983) El feminismo. Breviarios, 279. México: FCE.

Miguel, Jesús M. de y Carmen Domínguez-Alcón (1983) El mito de la Inmaculada Concepción. Col. La educación sentimental, 13. México: Anagrama.

MitCHELL, Juliet (1983) La liberación de la mujer: la larga lucha. Cuadernos Anagrama, 100. México: Anagrama.

Rowвотнам, Sheila (1983) «La mujer ignorada por la historia». En Tribuna feminista, 14. México: Debate.

- (1983a) «Mundo de hombre, conciencia de mujer». En Tribuna feminista, 4. México: Debate. 


\section{Bibliografía}

ACEVEDO, Marta (1970) «Nuestro sueño está en escarpado lugar (crónica de un miércoles santo entre mujeres)». En Siempre. La cultura en México, 451. 30 de septiembre. México.

- (1972) «Ni rosa ni azul». En Claudia, 84. México.

ALCALDE, Carmen (1981) Como leer un periódico. Col. Textos del periodismo. Barcelona: ATE.

BARDIN, Laurence (1977) L'analyse de contenu. París: PUF.

Bussy Genevois, Danièle et al. (1989) Discours de la presse, 22. Francia: Presses Universitaires de Rennes.

CAMACHO, Leonora (1977) «La mujer y el trabajo productivo en México». En Historia y sociedad. Vol. 4, núm. 14. México, D.F.

CERVANTES, Erika (2003) «Hacedoras de la historia, Griselda Álvarez, las pioneras de la política». En Cimac. 14 de octubre, México, D.F. CICAM, Centro de Investigación y Capacitación de la Mujer (1992)

Entrevista con la directora Ximena Bedregal Sáez. Julio. México, D.F.

Colectivo VenSeremos (1988) Entrevista con Anabela Rodrigo. 10 de junio. México, D.F.

- (2004) Entrevista electrónica con Ana Cristina Ramírez Barreto. Mayo.

CORREA, S. y R. Petchesky (1994) «Reproductive and sexual rights: a feminist perspective». En G. Sen, A. Germain y L.C. Chen (eds.) Population policies reconsidered. Health, empowerment and rights. Boston: Harvard University Press.

DELPHY, Christine (2004) «Retrouver l'élan du féminisme». En Le Monde diplomatique. Mayo.

ENTREVISTA con Josefina María Cendejas (2004) Colaboradora de La Boletina. Junio.

FEM (1983) «Taller del Chopo». Vol. VII, núm. 26. Febrero-marzo.

- (1988) Año 11, núm. 61.

GómEz Maganda de Anaya, Guadalupe (coord.) (1985) Decenio de la mujer ONU. Metas y logros de México en el decenio de la mujer, 19761985. ONU-Consejo Nacional para la Participación de la Mujer.

GRUPO Cinco: APIS-CIDHAL-EMAS-GEM-MAS (1991) Sobre el aborto (una antología). Mayo. México. 
GuBIN, Eliane et al. (dirs.) (2004) Le siècle des féminismes. París: Ed. de l'Atelier.

HERITIER, Françoise (1996) Masculin/féminin. La pensée de la différence. París: Odile Jacob.

HirATA, Helena et al. (2000) Dictionnaire cririque du fémnisme. París: PUF.

HIRIART, Berta (1984) «Cine de Mujeres». En Fem. Vol. IX, núm. 33. Abril-mayo, México, D.F.,

- (1991) «Romper el silencio». En Mujeres mexicanas en movimiento. Otro modo de ser. México: Centro de Comunicación Alternativa Alaíde Foppa.

ILET, Instituto Latinoamericano de Estudios Transnacionales (1984) Colectivo VenSeremos Michoacán, México. Unidad de comunicacion alternativa de la mujer. Santiago, Chile.

LA Boletina (1982) Núm. 1, año 1. Junio. México, D.F.

- (1982a) Núm. 2, año 1. Noviembre. México, D.F.

- (1983) Núm. 3, año 2. Enero. Morelia, Michoacán.

- (1983a) Núm. 4, año 2. Marzo. Morelia, Michoacán.

- (1983b) Núm. 5, año 2. Septiembre. Morelia, Michoacán.

- (1984) Núm. 6-7, año 3. Enero-abril. Morelia, Michoacán.

LAMAS, Marta (1994) «Algunas características del movimiento feminista en ciudad de México». En Mujeres y participación política. Avances y desafíos en América Latina. Bogotá: Editores T/M.

LAU Jaiven, Ana (1987) La nueva ola del feminismo. México: Planeta.

LUDEC, Nathalie (1992) La presse contemporaine pour femmes au Mexique. Tesis de doctorado. Francia: Universidad de Lille III.

- (2000) «Reconstruir la memoria de Alaíde Foppa a partir de su participación en la Red». En Debate feminista. Año II, vol. 22. Octubre. México D.F.

MonsIVÁIS, Carlos (2000) Aires de familia. Barcelona: Anagrama.

MEDIos Publicitarios SA de CV (1988) Medios impresos, tarifas y datos. Febrero. México, D.F.

NAVAS Turcios, María Candelaria et al. (1987) Feminismo y sectores populares en América Latina. Septiembre. México: EMAS/CIDHAL/ GEM/MAS/CEM/COVAG/APIS. 
OPus Gay (2004) Santiago de Chile. http://www.opusgay.cl/1315/article-29642.html

PERROT, Michelle (1998) Les femmes ou les silences de l'histoire. París: Flammarion.

SANTA Cruz, Adriana (1984) «Apuntes para una evaluación de la década. Mujer y comunicaciones en el marco latinoamericano». En Investigación sobre la mujer e investigación feminista: balance y perspectivas de la década de la mujer en América Latina. Diciembre. Montevideo.

- y Viviana Erazo (1980) COMPROPOLITAN, el orden transnacional y su modelo femenino. Un estudio de las revistas femeninas en América Latina. México: ILET/Nueva Imagen.

- (1983) «La comunicación alternativa de la mujer». En Revista de Ciencias Sociales, 25. Marzo.

SAU, Victoria (1981) Un diccionario ideológico feminista. Barcelona: Icaria.

TuÑón, Pablos (1987) Julia, Mujeres en México. Col. Mujeres en su tiempo. México: Planeta.

Fecha de recepción: 15/04/05. Aceptación: 07/06/2005. 